\title{
Telerradiografias obtidas em posição natural da cabeça alteram as grandezas cefalométricas?
}

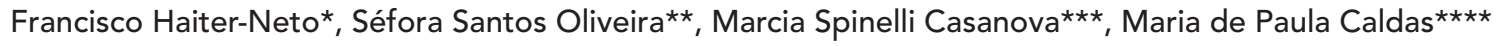

Resumo

Objetivo: o objetivo deste estudo foi comparar a real variação entre as medidas cefalométricas realizadas em telerradiografias laterais de um mesmo indivíduo, obtidas em posição natural da cabeça (PNC) e na técnica padrão, onde o plano de Frankfurt foi posicionado paralelamente ao solo. Foi realizada a análise facial, comparando as medidas de postura, perfil facial e medidas ântero-posteriores. Metodologia: a amostra foi composta por 50 telerradiografias laterais de indivíduos brasileiros, sendo 25 do gênero feminino e 25 do gênero masculino, com idade média de 21 anos. As medidas cefalométricas avaliadas foram divididas em três campos: 4 medidas de postura, 3 medidas de perfil e 9 medidas ântero-posteriores das bases ósseas. Resultados e Conclusão: para as medidas de postura, não foram encontradas diferenças significativas no grupo feminino, sendo que no grupo masculino foram encontradas diferenças significativas para os planos SN, Frankfurt, e Mandibular em relação à linha vertical verdadeira. Para as medidas ântero-posteriores e de perfil facial, não houve diferenças para ambos os grupos. Houve correlação do plano SN/V (Sela-Násio/Linha vertical verdadeira) com o plano palatino e Plano Horizontal de Frankfurt nas radiografias padrão e PNC, com exceção do grupo masculino nas telerradiografias laterais padrão. Não houve correlação entre o plano da base do crânio $\mathrm{SN} / \mathrm{V}$ com o plano mandibular em nenhuma das duas posições.

Palavras-chave: Cefalometria. Ortodontia. Radiografia.

\section{INTRODUÇÃO}

O sucesso do tratamento ortodôntico está relacionado com um correto diagnóstico. As complicações durante a mecânica ortodôntica originam-se de falhas e erros na realização do plano de tratamento. Em alguns pacientes, a informação cefalométrica não condiz com a realidade do caso. Além disso, diversas definições de pontos cefalométricos incluem termos diretamente re- lacionados com a orientação da cabeça do paciente, cujo posicionamento pode variar durante a obtenção da radiografia, podendo estar, por exemplo, em posição natural da cabeça (PNC) ou, mais comumente, com o plano de Frankfurt paralelo ao solo.

O interesse clínico na postura natural da cabeça (PNC) provém de estudos que correlacionam a morfologia craniofacial, a tendência de

* Professor Titular da Faculdade de Odontologia de Piracicaba, Universidade Estadual de Campinas - São Paulo, Brasil.

** Professora Adjunta da Faculdade de Odontologia de Itajaí, Univesidade do Vale do Itajaí - Santa Catarina, Brasil.

*** Doutoranda em Radiologia Odontológica pela Faculdade de Odontologia de Piracicaba, Universidade Estadual de Campinas - São Paulo, Brasil.

$\star \star \star \star$ Mestranda em Radiologia Odontológica pela Faculdade de Odontologia de Piracicaba, Universidade Estadual de Campinas - São Paulo, Brasil. 
crescimento futuro e as necessidades respiratórias. Acredita-se que os pontos de referência anatômicos intracranianos não são pontos estáveis. Baseados nesta afirmação, estudos sugerem o uso da PNC e da linha vertical verdadeira para a realização das análises cefalométricas ${ }^{5,11}$. A linha vertical verdadeira é a imagem radiográfica de um dispositivo externo com uma corrente de metal acoplada à haste do apoio frontal do cefalostato (Fig. 1). A importância em realizar registros cefalométricos e radiografias em PNC deve-se ao fato de corrigir o perfil do paciente, tal como ele se apresenta frente à sociedade.

Estudos descritos na literatura têm demonstrado que a postura natural da cabeça é notavelmente reproduzivele $2,6,8,10,12,13,14,17$. Com isso, análises cefalométricas baseadas na PNC continuam válidas ${ }^{4}$. Todavia, Bister et al. ${ }^{1}$ afirmaram que, apesar de perfeitamente reproduzivel, o posicionamento em PNC deve seguir corretamente um protocolo e, por ser realizado por um único operador, este deve ser cuidadosamente treinado. O uso da PNC, tanto para avaliação radiográfica como para a clínica, utiliza uma linha de referência externa à vertical verdadeira, que é usada como base tanto para análises dos traçados das telerradiografias laterais, como de fotografias para o estudo do perfil facial.

O presente estudo teve como propósito comparar a análise facial realizada sobre telerradiografias laterais, uma de forma convencional e outra obtida com o paciente em PNC, a fim de avaliar se há variação entre as medidas cefalométricas obtidas a partir destas técnicas radiográficas.

\section{MATERIAL E MÉTODOS}

Participaram deste estudo 50 indivíduos leucodermas, sendo 25 do gênero masculino e 25 do gênero feminino, com idade média de 21 anos. Foram realizadas 100 telerradiografias em norma lateral da cabeça, sendo 50 em PNC e 50 com a técnica convencional (Plano Horizontal de Frankfurt paralelo ao solo).

Como critério de seleção, os pacientes não poderiam apresentar nenhuma deformidade dento- esquelética, congênita ou traumática, e nenhuma história anterior de cirurgia de cabeça ou pescoço. Foi observado o equilíbrio facial dos tecidos moles, independente se os indivíduos tivessem sido submetidos ou não a tratamento ortodôntico prévio. Foram realizadas duas radiografias laterais de cabeça para cada paciente, a primeira em PNC e a segunda em posição convencional. Em ambas, foi utilizada uma corrente de metal acoplada ao cefalostato, que serviu para indicar a linha vertical verdadeira (V). As radiografias deveriam estar tecnicamente perfeitas, sem distorções, de modo a permitir uma boa visualização das estruturas anatômicas ósseas e do tecido do perfil mole.

\section{Registro da PNC}

Foi utilizado o protocolo sugerido por Solow e Tallgren ${ }^{18}$. Para os registros cefalométricos na posição de pé, foram tomados alguns cuidados:

1) No trajeto do paciente da porta de entrada do setor de Radiologia até o aparelho de raios $\mathrm{x}$, foi observada a forma de caminhar do mesmo, sem que este soubesse, para não induzir a uma mudança da postura. A ele, foi solicitado que se posicionasse com os pés levemente afastados, conforme as marcas que se encontravam previamente no chão (apenas como orientação).

2) Foi pedido ao paciente que respirasse tranquilamente, ficasse relaxado, com os braços soltos ao lado do corpo, e fizesse movimentos de balanço com a cabeça, tanto para frente como para trás, alternadamente, a fim de se conseguir uma posição de auto-equilíbrio, como proposto por SiersbaekNielsen e Solow ${ }^{16}$.

3) Com o objetivo de obter um ponto de referência externo para auxiliar na obtenção do equilíbrio natural da cabeça, um painel vertical e móvel, com gravuras em diferentes alturas, foi montado em frente ao cefalostato, a uma distância de aproximadamente $190 \mathrm{~cm}$. Ao paciente, foi solicitado que escolhesse uma das figuras que estivesse à altura dos olhos e fixasse o olhar sobre ela. Neste momento, foi verificado se a pupila 
dos olhos encontrava-se no centro dos mesmos e o posicionador nasal foi colocado levemente sob a glabela. As hastes de ouvido foram posicionadas aproximadamente $1 \mathrm{~cm}$ à frente do tragus, em leve contato com a pele, tendo o cuidado de não modificar esta posição (Fig. 1). O paciente recebeu orientações para ficar com os dentes levemente ocluídos em máxima intercuspidação habitual e os lábios deviam se tocar sem nenhuma tensão.

4) Uma corrente de metal com um peso na ponta foi acoplada à haste do apoio frontal, para indicar a linha vertical verdadeira (V) $)^{15,16,18}$.

5) Após checar o item 3 e 4, o exame radiográfico foi realizado.

\section{Registro da projeção cefalométrica lateral- padrão}

A cabeça do paciente foi posicionada com o

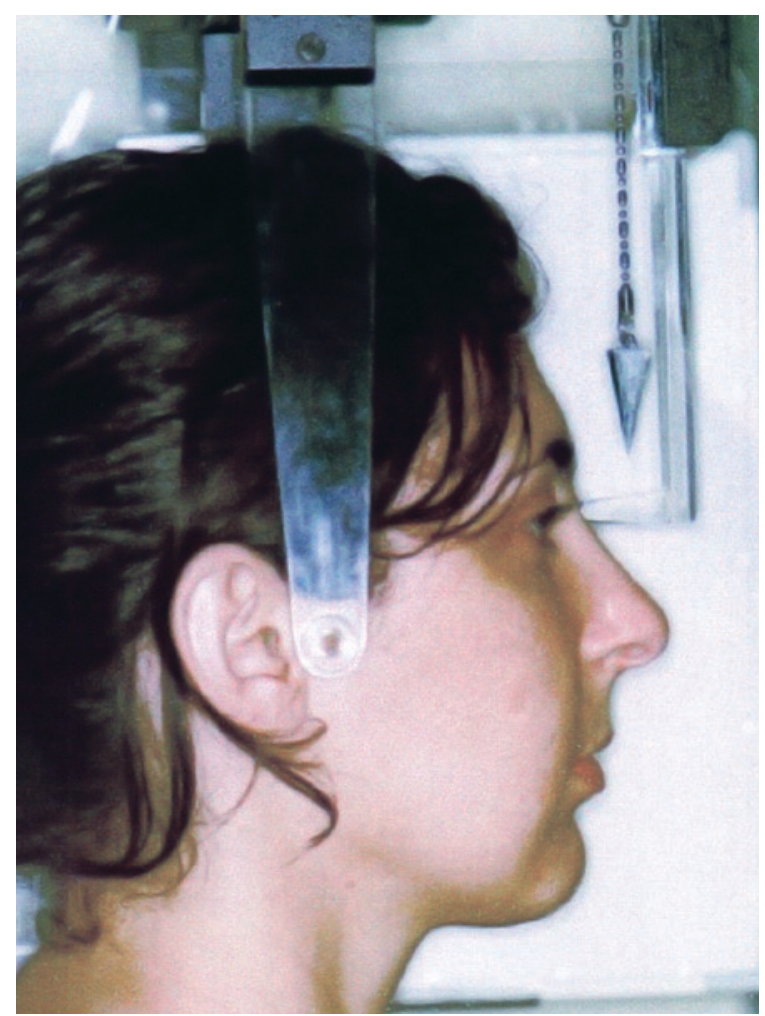

FIGURA 1 - Paciente posicionado em PNC. lado esquerdo da face próxima ao chassis e o plano sagital mediano paralelo ao plano do filme. As olivas foram colocadas no conduto auditivo externo e o apoio nasal foi posicionado na glabela, estando o plano horizontal de Frankfurt posicionado paralelamente ao solo (Fig. 2).

\section{Análises cefalométricas}

Depois de obtidas as radiografias, estas foram submetidas às análises cefalométricas, realizandose o traçado anatômico e marcados os pontos cefalométricos (Fig. 3).

Os pontos intracranianos utilizados foram os da análise cefalométrica de Steiner: Ponto A, Espinha Nasal Anterior (ENA), Espinha Nasal Posterior (ENP), Ponto B, Sela Túrcica $(\mathrm{S})$, Násio $(\mathrm{N})$, Orbital (Or), Pório (Po), Pogônio duro (Pog), Gônio (Go), Mentoniano (Me), Gnátio (Gn), Básio

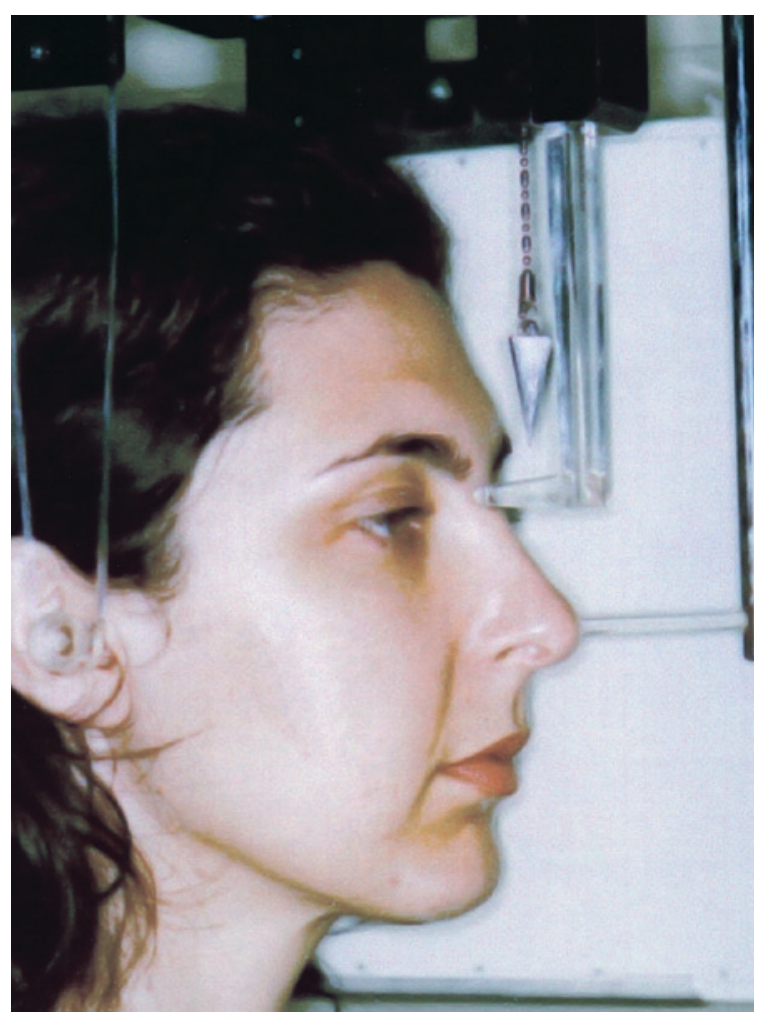

FIGURA 2 - Paciente em posição convencional. 


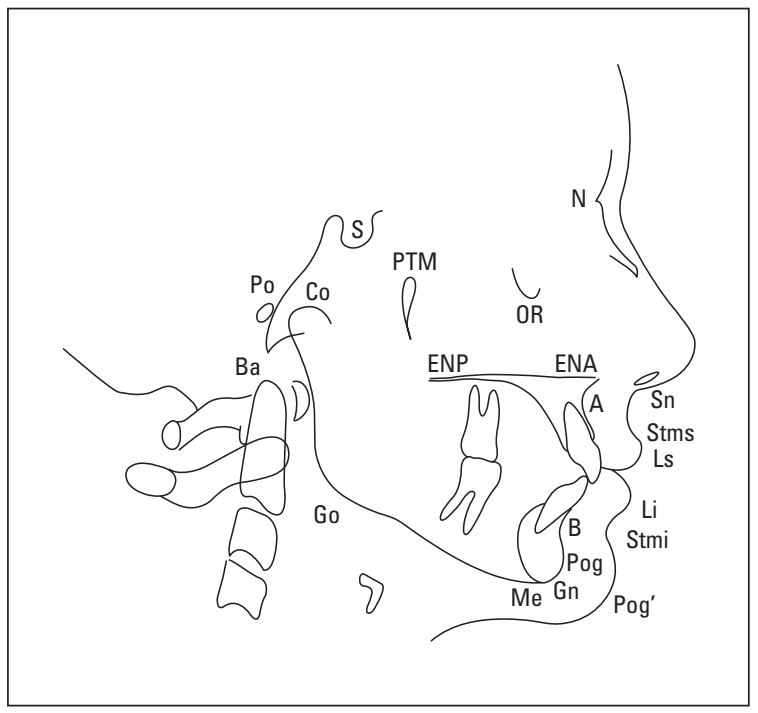

FIGURA 3 - Traçado anatômico e pontos cefalométricos.

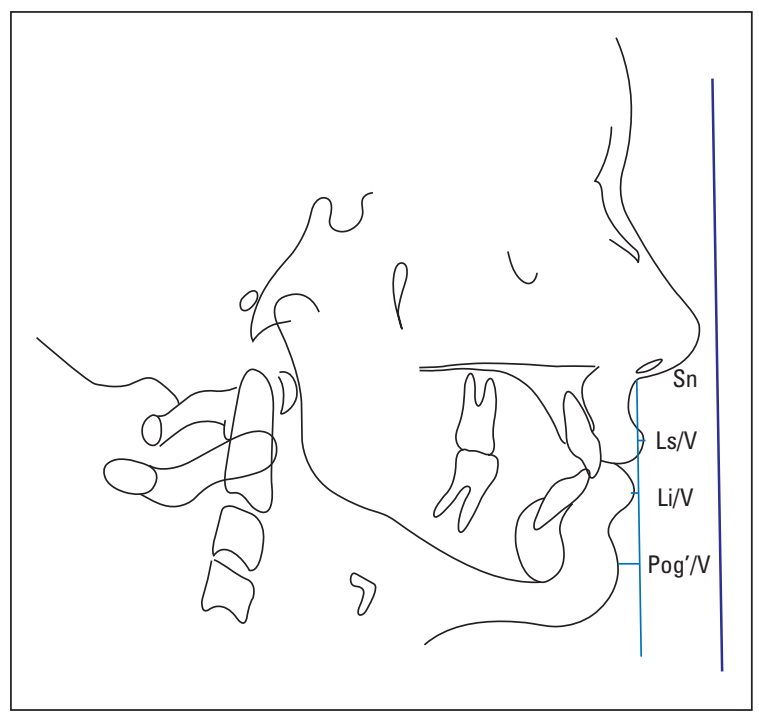

FIGURA 5 - Medidas do perfil facial.

(Ba), Pterigomaxilar (PTM) e Côndilo (Co).

Os pontos cefalométricos de tecidos moles foram marcados de acordo com a análise de Jacobson e Vlachos: Subnasal (Sn), Lábio superior (Ls), Stomion superior (Stms), Stomion inferior (Stmi), Pogônio mole (Pog').

Foram mensurados dezesseis fatores, divididos nos três campos descritos a seguir.

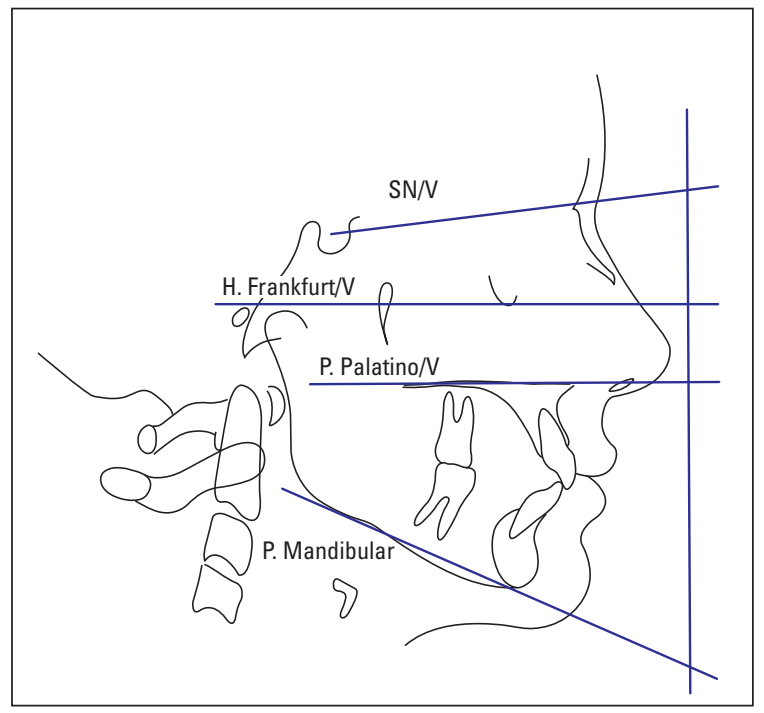

FIGURA 4 - Medidas da postura da cabeça.

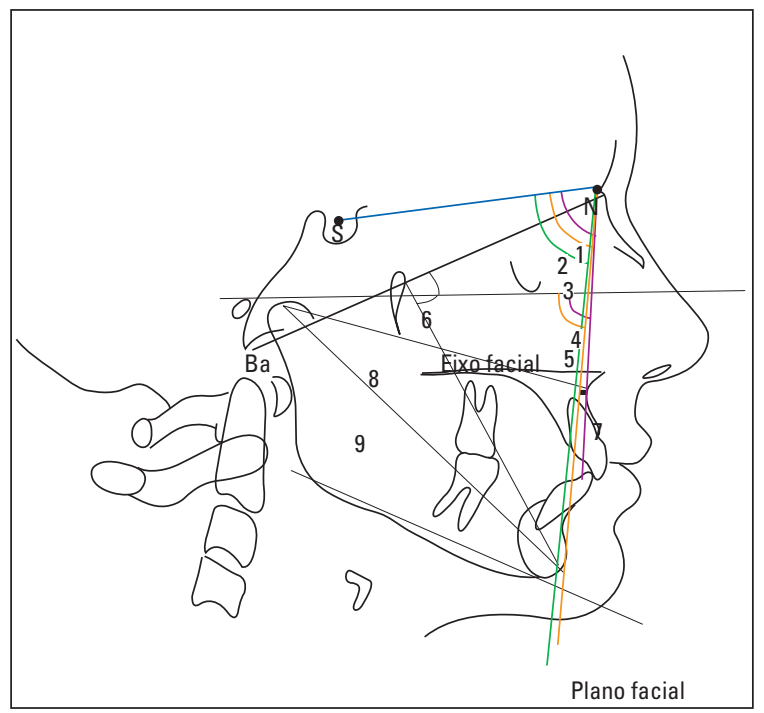

FIGURA 6 - Medidas ântero-posteriores: 1) SNA; 2) SNPog; 3) SNB; 4) profundidade maxilar; 5) profundidade facial; 6) ângulo do eixo facial; 7) convexidade do ponto $A$; 8) CoA; 9) CoGn.

1) Medidas da Postura da cabeça: SN/V, H. Frankfurt/V, P. Palatino/V, P. Mandibular (Fig. 4).

2) Medidas do Perfil Facial: Ls/V, Li/V, Pog'/V (Fig. 5).

3) Medidas ântero-posteriores: SNA, SNB, SN Pog, CoA, CoGn, Convexidade do Ponto A, Profundidade Facial, Eixo facial, Profundidade maxilar (Fig. 6). 
Após a obtenção das medidas cefalométricas, foi utilizado o teste $t$ pareado, com o objetivo de comparar as medidas encontradas nas telerradiografias obtidas em PNC com aquelas encontradas nas telerradiografias obtidas de forma convencional.

\section{RESULTADOS E DISCUSSÃO}

Analisando a tabela 1, observa-se que não foram encontradas diferenças estatisticamente significantes nas medidas das posturas obtidas em PNC e técnica convencional, para o gênero feminino. Esses resultados demonstram que as mulheres apresentam pouca alteração postural. Já na amostra masculina, foram encontrados valores estatisticamente diferentes para todas as medidas, com exceção de H. Frankfurt/V. Essa diferença encontrada para as medidas de postura entre homens e mulheres está de acordo com Cooke e $\mathrm{Wei}^{3}$, que confirmaram que os homens tendem a adotar uma postura mais relaxada na posição de autoequilíbrio (posição natural da cabeça), enquanto as mulheres, segundo os autores, são encorajadas socialmente a adotar uma postura correta.

Para as medidas de angulação da base do crânio em relação à vertical verdadeira (SN/V), foram encontradas medidas variando de $96,98^{\circ}$ a $98,94^{\circ}$, diferindo dos resultados apresentados por Showfety et al. ${ }^{15}$, que encontraram uma média de $81,2^{\circ}$ a $83^{\circ}$. Esta diferença pode ser justificada pelo fato da amostra usada por estes autores ter sido composta por indivíduos do gênero feminino com severa má oclusão, com indicação de cirurgia ortognática.

Em relação às medidas lineares do perfil facial (Tab. 2), novamente não houve diferenças estatisticamente significantes entre as duas técnicas, para o gênero feminino. Para o gênero masculino, apenas a medida Pog'/V apresentou diferença estatisticamente significante, possivelmente devido

Tabela 1 - Médias das medidas angulares, desvios-padrão e valores do teste t, obtidos nos cefalogramas traçados em postura da cabeça em PNC e na forma convencional, em ambos os gêneros.

\begin{tabular}{|c|c|c|c|c|c|c|c|}
\hline & \multirow{2}{*}{ posição } & \multicolumn{3}{|c|}{ feminino } & \multicolumn{3}{|c|}{ masculino } \\
\hline & & média & d.p. & $\mathbf{p}$ & média & d.p. & $\mathbf{p}$ \\
\hline \multirow{2}{*}{ SN V } & PNC & 98,73 & 5,15 & \multirow{2}{*}{0,7920} & 98,94 & 6,00 & \multirow{2}{*}{$0,0216^{*}$} \\
\hline & convencional & 98,91 & 4,19 & & 96,78 & 4,71 & \\
\hline \multirow{2}{*}{ H.Fr V } & PNC & 90,73 & 5,09 & \multirow{2}{*}{0,6570} & 90,84 & 5,83 & \multirow{2}{*}{0,0784} \\
\hline & convencional & 90,41 & 3,03 & & 89,18 & 4,42 & \\
\hline \multirow{2}{*}{ P. PI V } & PNC & 90,36 & 4,94 & \multirow{2}{*}{0,7056} & 91,10 & 5,53 & \multirow{2}{*}{$0,0085^{*}$} \\
\hline & convencional & 90,08 & 3,75 & & 88,90 & 3,85 & \\
\hline \multirow{2}{*}{ P. Md V } & PNC & 66,94 & 5,29 & \multirow{2}{*}{0,1664} & 69,68 & 7,60 & \multirow{2}{*}{$0,0385^{*}$} \\
\hline & convencional & 65,78 & 4,35 & & 68,18 & 6,66 & \\
\hline
\end{tabular}

PNC - Posição Natural da Cabeça.

* Significativo para o teste t bilateral $(p<0,05)$.

Tabela 2 - Médias das medidas lineares do Perfil Facial, obtidas nas radiografias em PNC e técnica convencional, em ambos os gêneros.

\begin{tabular}{|c|c|c|c|c|c|c|c|c|c|}
\hline & \multirow{2}{*}{ posição } & \multicolumn{4}{|c|}{ feminino } & \multicolumn{4}{|c|}{ masculino } \\
\hline & & amplitude & média & d.p. & p & amplitude & média & d.p. & p \\
\hline \multirow{2}{*}{$\mathrm{Ls} / \mathrm{V}$} & PNC & $+3,5$ a $-2,5$ & 0,1 & 1,72 & \multirow{2}{*}{0,6812} & $+3,5$ a $-2,5$ & 0,27 & 1,86 & \multirow{2}{*}{0,1219} \\
\hline & padrão & $+3 a-2,8$ & 0,01 & 1,79 & & $+2,5$ a $-3,5$ & $-0,24$ & 1,37 & \\
\hline \multirow{2}{*}{$\mathrm{Li} / \mathrm{V}$} & PNC & +4 a $-7,5$ & $-2,06$ & 2,89 & \multirow{2}{*}{0,8724} & $+4,5$ a $-4,5$ & $-1,47$ & 2,78 & \multirow{2}{*}{0,0919} \\
\hline & padrão & +3 a -9 & $-2,16$ & 2,92 & & +1 a $-5,5$ & $-2,39$ & 1,93 & \\
\hline \multirow{2}{*}{ Pog/V } & PNC & +6 a $-13,5$ & $-6,48$ & 5,29 & \multirow{2}{*}{0,2736} & +5 a $-13,5$ & $-6,36$ & 6,45 & \multirow{2}{*}{$0,0208^{*}$} \\
\hline & padrão & $+3,5$ a $-12,5$ & $-7,24$ & 4,08 & & $+5 a-20,5$ & $-8,4$ & 35,85 & \\
\hline
\end{tabular}

PNC - Posição Natural da Cabeça.

* Significativo para $\mathrm{p}<0,05$. 
Telerradiografias obtidas em posição natural da cabeça alteram as grandezas cefalométricas?

Tabela 3 - Médias das medidas ântero-posteriores, desvios-padrão e valores do teste t, obtidos nos cefalogramas traçados em postura da cabeça em PNC e na forma convencional, em ambos os gêneros.

\begin{tabular}{|c|c|c|c|c|c|c|c|}
\hline & \multirow{2}{*}{ posição } & \multicolumn{3}{|c|}{ feminino } & \multicolumn{3}{|c|}{ masculino } \\
\hline & & média & d.p. & p & média & d.p. & $\mathbf{p}$ \\
\hline \multirow{2}{*}{ SNA } & PNC & 80,74 & 3,09 & \multirow{2}{*}{0,4373} & 82,32 & 4,55 & \multirow{2}{*}{0,4240} \\
\hline & convencional & 80,52 & 2,84 & & 81,98 & 4,57 & \\
\hline \multirow{2}{*}{ SNB } & PNC & 77,98 & 3,61 & \multirow{2}{*}{0,8792} & 80,38 & 4,28 & \multirow{2}{*}{$0,0331 *$} \\
\hline & convencional & 78,04 & 3,11 & & 79,02 & 4,12 & \\
\hline \multirow{2}{*}{ SN-Pog } & PNC & 78,88 & 4,09 & \multirow{2}{*}{0,0739} & 81,20 & 4,33 & \multirow{2}{*}{0,3395} \\
\hline & convencional & 78,04 & 3,11 & & 80,82 & 4,47 & \\
\hline \multirow{2}{*}{$\mathrm{CoA}$} & PNC & 88,82 & 3,95 & \multirow{2}{*}{0,2601} & 94,40 & 4,85 & \multirow{2}{*}{0,4793} \\
\hline & convencional & 89,20 & 3,46 & & 94,06 & 4,56 & \\
\hline \multirow{2}{*}{ CoGn } & PNC & 116,88 & 5,13 & \multirow{2}{*}{0,3385} & 125,84 & 5,37 & \multirow{2}{*}{0,7098} \\
\hline & convencional & 117,20 & 5,12 & & 126,00 & 6,47 & \\
\hline \multirow{2}{*}{ Conv.A } & PNC & 1,24 & 2,83 & \multirow{2}{*}{0,2568} & 0,64 & 3,61 & \multirow{2}{*}{0,0919} \\
\hline & convencional & 1,50 & 3,04 & & 0,32 & 3,48 & \\
\hline \multirow{2}{*}{ P. Facial } & PNC & 87,60 & 4,82 & \multirow{2}{*}{0,7044} & 88,74 & 3,93 & \multirow{2}{*}{0,7737} \\
\hline & convencional & 87,72 & 3,96 & & 88,84 & 3,46 & \\
\hline \multirow{2}{*}{ E. Facial } & PNC & 88,86 & 4,28 & \multirow{2}{*}{0,2911} & 92,12 & 5,22 & \multirow{2}{*}{0,7601} \\
\hline & convencional & 89,32 & 4,82 & & 92,18 & 5,34 & \\
\hline \multirow{2}{*}{ P. Maxilar } & PNC & 88,82 & 3,60 & \multirow{2}{*}{0,5313} & 89,76 & 4,11 & \multirow{2}{*}{0,8430} \\
\hline & convencional & 89,08 & 2,49 & & 89,68 & 3,85 & \\
\hline
\end{tabular}

PNC - Posição Natural da Cabeça.

* Significativo $\mathrm{p}<0,05$.

às alterações nas medidas de postura relativas ao plano mandibular. Esse fato também foi demonstrado por Lundstrom e Cooke ${ }^{9}$ e Lundstron et $\mathrm{al}^{7}$, que encontraram diferenças significativas entre os gêneros, ao estudarem a análise proporcional do perfil facial.

Ao avaliarmos as medidas ântero-posteriores da maxila e mandíbula (Tab. 3), observa-se ausência de diferenças estatisticamente significantes entre as medidas obtidas em PNC e pela técnica convencional, em ambos os gêneros. Isso pode ser explicado devido à pequena modificação postural encontrada, o que não resultou em modificação significativa na inclinação do plano de Frankfurt, e pelo fato da amostra utilizada não apresentar discrepâncias dentoalveolares ou esqueléticas. Isso se torna extremamente relevante ao verificar na literatura a afirmação feita por TNG et al. ${ }^{19}$ de que alterações de $\pm 10^{\circ}$ na inclinação do plano de Frankfurt podem produzir efeitos significativos sobre os valores cefalométricos.

\section{CONCLUSÃO}

Apesar da amostra do presente estudo constar de pacientes jovens com harmonia facial, para o gênero masculino foram encontradas diferenças relacionadas às medidas de posturas e ao perfil facial. Deste modo, consideramos que a PNC deve ser utilizada para análise cefalométrica lateral, que, em adição à avaliação clínica, permite um planejamento mais seguro e mais próximo da realidade do paciente, visto que este tipo de avaliação respeita a postura assumida pelo indivíduo em seu cotidiano.

Enviado em: outubro de 2005 Revisado e aceito: fevereiro de 2006 
Are there cephalometric measurements alterations using natural head position?

\begin{abstract}
Aim: the aim of this study was to quantify the real variation of cephalometric measurements obtained from lateral cephalometric radiographs using a natural head posture (NHP) and a conventional technique, positioning Frankfurt Horizontal Plane parallel to the horizontal. A facial analysis was performed in order to compare postural measurements, facial profile and antero-posterior measurements. Methods: fifty lateral cephalometric radiographs from 25 females and 25 males composed the sample, mean aged 21 years. The cephalometric measurements were divided into three groups: 4 postural measurements, 3 profile measurements, and 9 antero-posterior measurements. Results and Conclusion: no statistically significant differences were found for females, while males showed statistically significant differences for sella-nasion (SN), Frankfurt, and mandibular planes in relation to true vertical line (T). For antero-posterior and facial profile measurements, no statistically significant differences were found in both groups. A correlation of the SN/V (sella-nasion/true vertical line) plane with the palatal and Frankfurt planes was found for both techniques, except when conventional lateral cephalometric radiographs were taken from males. No correlation was found between the SN/V base of the skull plane and the mandibular plane in both positions.
\end{abstract}

Key words: Cephalometry. Orthodontics. Radiography.

\title{
REFERÊNCIAS
}

1. BISTER, D. et al. Natural head posture-considerations of reproducibility. Eur. J. Orthod., Oxford, v. 24, no. 5, p. 457-470, 2002.

2. CHIU, C. S.; CLARK, R. K. Reproducibility of natural head position. J. Dent., Bristol, v. 19, no. 2, p. 130-131, 1991

3. COOKE, M. S.; WEI, S. H. The reproducibility of natural head posture: a methodological study. Am. J. Orthod. Dentofacial Orthop., St. Louis, v. 93, no. 4, p. 280-288, 1988.

4. GOEL, S.; BANSAL, M.; KALRA, A. A preliminary assessment of cephalometric orthodontic superimposition. Eur. J. Orthod., Oxford, v. 26, no. 2, p. 217-222, 2004.

5. GUIMARÃES, M. A. C.; RAMALHO, S. A. A influência da postura da coluna cervical, vista em norma lateral, na posição natural da cabeça (PNC) para diagnóstico ortodôntico. RGO, Porto Alegre, v. 51, n. 4, p. 265-272, 2003.

6. LUIK, N. H. et al. The reproducibility of the natural head position in lateral cephalometry radiographs. Br. J. Oral Maxillofac. Surg., Edinburgh, v. 24, no. 5, p. 357-366, 1986.

7. LUNDSTROM, A. et al. A proportional analysis of the soft tissue facial profile in young adults with normal occlusion. Angle Orthod., Appleton, v. 62, no. 2, p. 127-133, 1992.

8. LUNDSTROM, A. et al. Natural head position and natural head orientation: basic considerations in cephalometric analysis and research. Eur. J. Orthod., Oxford, v. 17, no. 2, p. 111-120, 1995.

9. LUNDSTROM, A.; COOKE, M. Proportional analysis of the facial profile in natural head position in Caucasian and Chinese children. Br. J. Orthod., Oxford, v. 18, no. 1, p. 43-49, 1991.

10. LUNDSTROM, F.; LUNDSTROM, A. Natural head position as a basis for cephalometric analysis. Am. J. Orthod. Dentofacial Orthop., St. Louis, v. 101, no. 3, p. 244-247, 1992.

11. MARTON, N.; MARTINS, N. S. Estudo comparativo das radiografias cefalométricas laterais em posição convencional e em postura natural da cabeça (postura preconizada por Rocabado) uma análise dos arcos dentários. Ortodontia, São Paulo, v. 34, n. 2, p. 73-79, 2001

12. NEGREIROS, P. E.; SIQUEIRA, V. C. V. O efeito da alteração da posição natural da cabeça (PNC) sobre as medidas cefalométricas. Rev. Dental Press Ortodon. Ortop. Facial, Maringá, v. 9, n. 3, p. 59-76, 2004.

13. PENG, L.; COOKE, M. S. Fifteen-year reproducibility of natural head posture: a longitudinal study. Am. J. Orthod. Dentofacial Orthop., St. Louis, v. 116, no. 1, p. 82-85, 1999.
14. RINO-NETO, J. et al. Avaliação da reprodutibilidade da posição natural da cabeça: estudo radiográfico. Ortodontia São Paulo, n. 4, p. 55-68, 2002.

15. SHOWFETY, K. J. et al. Associations between the postural orientation of sella-nasion and skeletodental morphology. Angle Orthod., Appleton, v. 57, no. 2, p. 99-112, 1987.

16. SIERSBAEK-NIELSEN, S. B.; SOLOW, B. Intra- and interexaminer variability in head posture recorded by dental auxiliaries. Am. J. Orthod., St. Louis, v. 82, no. 1, p. 50-57, 1982.

17. SOLOW, B.; SONNESEN, L. Head posture and malocclusions. Eur. J. Orthod., Oxford, v. 20, no. 6, p. 685-693, 1998.

18. SOLOW, B.; TALLGREN, A. Natural head position in standing subjects. Acta Odont. Scand., Stockholm, v. 29, no. 5, p. 591607, 1971.

19. TNG, T. T. et al. Effect of head posture on cephalometric sagittal angular measures. Am. J. Orthod. Dentofacial Orthop., St. Louis, v. 101, no. 4, p. 337-341, 1993.

Endereço para correspondência

Francisco Haiter Neto

Av. Limeira, 901 - Bairro Areião - Cx. Postal 52

CEP: 13414-901 - Piracicaba/São Paulo

E-mail: haiter@fop.unicamp.br 